\title{
Conditional Quantitative Trait Locus Mapping of Wheat Seed Protein-fraction in Relation to Starch Content
}

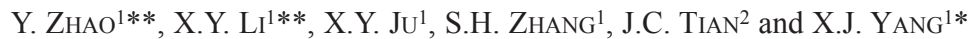 \\ ${ }^{1}$ Huabei Key Laboratory of Crop Germplasm, Hebei Agricultural University, Baoding 071000, Hebei, China \\ ${ }^{2}$ Shandong Agricultural University, Tai'an 271018, China
}

(Received 12 July 2016; Accepted 26 January 2017; Communicated by R.N. Chibbar)

\begin{abstract}
Protein and starch are important in wheat quality and yield. To understand the genetic relationship between protein and starch at the quantitative trait locus (QTL)/gene level, 168 doubled haploid (DH) lines were used at three locations over 2 years. The QTLs for proteinfraction contents and starch content were analyzed by unconditional and conditional QTL mapping. We detected 17 unconditional additive QTLs (four albumin QTLs, three globulin QTLs, six gliadin QTLs, four glutenin QTLs) controlling protein-fraction contents. We detected 19 conditional QTLs (five albumin QTLs, three globulin QTLs, five gliadin QTLs, six glutenin QTLs) based on starch content. Of these QTLs, QAlu1B, QGlo6A, QGli1B, QGli7A, QGlu1B and $Q G l u 1 D$ increased the protein-fraction contents independent of the starch content. These QTLs could regulate the usual inverse relationship between protein and starch in wheat seeds. The results could possibly be used in the simultaneous improvement of grain protein and starch content in wheat breeding.
\end{abstract}

Keywords: wheat (Triticum asetivum L.), protein-fraction contents, starch content, unconditional QTL, conditional QTL

\section{Introduction}

Protein and starch are the principal components of wheat endosperm. Proteins are the most important wheat grain components governing end-use quality (Weegels et al. 1996). On the basis of their solubility properties, wheat grain proteins are traditionally classified into albumins, globulins, gliadins, and glutenins (Osborne et al. 1907). Albumins (accounting for $3 \%$ to $5 \%$ of total wheat protein) and globulins (accounting for $6 \%$ to $10 \%$ of total wheat protein) are readily soluble in water and in salt solution, respectively (Pence et al. 1954; Singh and Skerritt 2001; Lian et al. 2014). Compared with other wheat grain proteins, albumins and globulins are considered to have nutritionally superior amino acid compositions because of their higher lysine and methionine contents (Lasztity 1984).

Gliadins (accounting for $40 \%$ to $50 \%$ of total wheat protein) and glutenins (accounting for $30 \%$ to $40 \%$ of total wheat protein) can be extracted individually from flour using a solution of alcohol and dilute acid or alkali, respectively (Huebner and Wall 1976; Pike

\footnotetext{
*Corresponding author; E-mail: shmyxj@126.com; Phone: +86-0312-7528260; Fax: +86-0312-7528265;

$* *$ Both authors contributed equally to this work.
} 
and MacRitchie 2004; Lian et al. 2014). Hydrated gliadins have little elasticity and are less cohesive than glutenins; they contribute mainly to dough viscosity and extensibility (Wieser 2007). In wheat flour dough, baking performance strongly depends on the molecular weight distribution of glutenins. Glutenins appear to be largely responsible for gluten elasticity (MacRitchie 1992; Wieser 2007). Gliadins and glutenins are important contributors to processing quality, as they are involved in the formation of the gluten network (Branlard and Dardevet 1985).

Starch plays a crucial role in determining the wheat grain yield (Jenner 1994; Hurkman et al. 2003), and also affects the flour processing quality (Yamamori and Quynh 2003; Yamamori 2009). Several studies on the significant negative correlation between starch and wheat flour processing quality have been reported (Martin et al. 2004; Zhang et al. 2009). Although extensive QTL analysis of the protein-fraction contents and starch content of wheat has been conducted, less information is available about the genetic relationship of these components at the QTL/gene level: to date, no studies have investigated this relationship.

Since Zhu (1995) proposed conditional analysis methods to exclude the contribution of a causal trait to the variation of the resultant trait, conditional QTL mapping methods have been developed. Once the contribution of a causal trait has been excluded, the remaining variation of the resultant trait is defined as conditional variation, or net variation, which indicates the extra effects of genes that are independent of the causal trait (Liu et al. 2008). This method was first applied to the studies on QTL of developmental behavior in rice (Cheng et al. 2013), maize (Osman et al. 2013), cotton (Ye et al. 2003), soybean (Jiang et al. 2010; Han et al. 2011), wheat (Liu et al. 2011; Zhu et al. 2011; Jiang et al. 2013). Several studies on the analysis of multiple related traits in conditional QTL mapping methods (Zhao et al. 2006; Wang et al. 2012), defining the genetic relationship among different traits at the QTL level.

Our objective was to dissect the genetic relationship between protein-fraction contents and starch content using unconditional and conditional QTL mapping analyses. By comparing unconditional and conditional QTLs, the genetic inter dependencies between protein-fraction contents and starch content can be identified at the level of the individual QTL. This comparison may provide valuable information for marker-assisted selection to improve protein without reducing starch.

\section{Materials and Methods}

\section{Plant materials}

For linkage map construction, we used a population of $168 \mathrm{DH}$ lines derived from a cross between Chinese wheat cultivars Huapei 3 and Yumai 57. Huapei 3 was released by Henan Province, China, in 2006 (Hai and Kang 2007) and Yumai 57 was released nationally in 2003 (Guo et al. 2004). The two parents, which are cultivated over a large area of the Yellow and Huai valley wheat region of China, differ with respect to several agro- 
nomically important traits, as well as traits related to baking quality (Guo et al. 2004; Hai and Kang 2007; Zhao et al. 2010).

\section{Experimental design}

The 168 DH lines and their parents were sown in 2011 and 2012 (for harvest in 2012 and 2013 ) in Hebei Agricultural University (Baoding $38.85^{\circ} \mathrm{N}, 115.5^{\circ} \mathrm{E}$ ), Xingtai Agricultural Institute (Xingtai $37.13^{\circ} \mathrm{N}, 114.68^{\circ} \mathrm{E}$ ), and Cangzhou Agricultural Institute (Cangzhou $38.58^{\circ} \mathrm{N}, 116.82^{\circ} \mathrm{E}$ ), China. The experimental design followed a randomized complete block design with three replications. Plants were grown under normal field conditions in three-row plots ( $3 \mathrm{~m}$ long, with between-row spacing of $25 \mathrm{~cm}$ ), with 100 seeds sown in each row. The date of anthesis was recorded for each material. Six plants of each experimental line, chosen from the middle of the inner row, were harvested at maturity. Ears of the harvested plants were dried until the seed mass was stable. The seeds were extracted from the dry ears by hand, milled into wholemeal flour using a Model 3100 hammer mill (Perten Instruments, Hägersten, Sweden), and passed through an 80-mesh sieve.

\section{Trait evaluation}

Wheat protein-fractions were prepared from wholemeal flour according to the method described by George and Scott (1995) with minor modifications. The milled wheat flour $(0.1 \mathrm{~g})$ was sequentially extracted with $50 \mathrm{mM}$ phosphate buffer $(\mathrm{pH}$ 6.8) for albumin. The same buffer containing $0.1 \mathrm{M} \mathrm{NaCl}$ was used for globulin; 70\% aqueous ethanol was used for gliadin; $0.05 \mathrm{M} \mathrm{NaOH}$ was used for glutenin. The sample was stirred with $10 \mathrm{~mL}$ of solvent at room temperature for $30 \mathrm{~min}$. Extracts were separated from residues by centrifugation (4000 rpm for $10 \mathrm{~min}$ ). The procedure was repeated three times. The extracts were stored in a freezer for further analysis. The protein content of extracts was measured by the semi-micro-Kjeldahl method (Chinese Bureau of Standardization 1982) using a Kjeltec 2100 Auto-analyser (Foss AB, Hägersten, Sweden). A nitrogen conversion factor of 5.7 was used to compute the protein value. The starch content of the flour was assayed according to the method described by Holm et al. (1986) using a UV-240 UV/V spectrophotometer (Shimadzu, Tokyo, Japan).

\section{Data analyses}

Basic statistical analysis was carried out using SPSS version 17.0 (SPSS, Chicago, IL, USA). The genetic linkage map consisted of 368 markers: SSR loci, EST loci, intersimple sequence repeat locus, and HMW-GS locus. The map covered a total length of $3074.1 \mathrm{cM}$, with an average distance of $8.35 \mathrm{cM}$ between adjacent markers. These linked markers formed 24 linkage groups over 21 chromosomes (Zhang et al. 2008; Zhao et al. 2010; Li et al. 2012). Genomic locations of marker loci/linkage groups were determined based on the wheat consensus map constructed by Somers et al. (2004). In our case, the 
chromosomal locations of 30 loci $(7.03 \%)$ were different from those on the Somers et al. (2004) map.

Both unconditional and conditional QTLs were detected under a mixed linear model using IciMapping v3.2 (http://www.isbreeding.net/). Composite interval analysis was performed by forward-backward stepwise multiple linear regression, with a probability into and out of the model of 0.05 and a window size of $10 \mathrm{cM}$. For each data set, significant thresholds for QTL detection were calculated using 1000 permutations and genomewide error rates of 0.10 (suggestive) and 0.05 (significant).

Conditional genetic analysis was conducted based on the phenotypic values of the protein-fractions conditioned on the starch content. Conditional phenotypic values $\mathrm{y}(\mathrm{T} 1 \mid \mathrm{T} 2)$ were obtained by the mixed-model approach (Zhu 1995; Wen and Zhu 2005) for the conditional analysis of quantitative traits described by Zhu (1995), where T1|T2 means trait 1 conditioned on trait 2 (for example, Alu|starch refers to protein-fraction contents conditioned on starch content).

The software QGAStation 1.0 (http://ibi.zju.edu.cn/software/qga/) was used to determine the conditional phenotypic values $\mathrm{y}(\mathrm{T} 1 \mid \mathrm{T} 2)$ as input data for conditional QTL mapping, which used the composite interval mapping method. QTLs were designated using the format "QTL + trait + chromosome" in accordance with international nomenclature for QTLs in wheat and related species (McIntosh et al. 1994). QTLs detected through unconditional and conditional QTL mapping methods are called unconditional and conditional QTLs, respectively, in the present paper.

\section{Results}

\section{Phenotypic variation and correlations between traits}

Phenotypic values of the content of wheat grain protein-fractions and starch across six environments are shown in Table $\mathrm{S} 1 *$. The $\mathrm{DH}$ population displayed a wide range of variation. Frequency distributions of evaluated traits showed continuous variation and significant transgressive segregation in both directions, indicating that these traits were under polygenic control and suitable for QTL analysis. Correlation analyses were performed between protein-fraction content and starch content in different environments (Table S2). The correlation coefficients relating the protein-fraction contents and the starch content were significant $(P \leq 0.05)$ and negative for all proteins except albumin, which was positive.

\section{QTL mapping}

\section{Unconditional and conditional QTLs for albumin content}

A total of four unconditional additive QTLs for albumin content were identified across 2 years at three locations (Table S3; Fig. S1). These QTLs were mapped to chromosomes

\footnotetext{
*Further details about the Electronic Supplementary Material (ESM) can be found at the end of the article.
} 
$1 \mathrm{~B}, 3 \mathrm{~A}$, and $6 \mathrm{~A}$ and accounted for $6.16-18.36 \%$ of the phenotypic variation. Three major QTLs explained more than $10 \%$ of the phenotypic variation: QAlu1B, QAlu1B-1 and QAlu6A. QAlu1B was detected at three environments and had the largest contribution to the phenotypic variation. It accounted for $8.13-18.36 \%$ of the phenotypic variation.

Four conditional additive QTLs for albumin content were identified (Table S4; Fig. S1). These QTLs were mapped to chromosomes 1B, 6A and 6B and accounted for $8.63-17.24 \%$ of the phenotypic variation. Among them, QAlu1B was detected at three environments and accounted for $8.15-17.24 \%$ of the phenotypic variation. Two major QTLs each explained more than $10 \%$ of the phenotypic variation: QAlu $1 B$ and QAlu1B-2. $Q A l u 1 B$ had the largest contribution to the phenotypic variation (17.24\%).

\section{Unconditional and conditional QTLs for globulin content}

Three different unconditional additive QTLs for globulin content were identified within 2 years at three locations (Table S3; Fig. S1). These QTLs were mapped to chromosomes $1 \mathrm{~B}, 6 \mathrm{~A}$ and $6 \mathrm{~B}$ and accounted for $9.34-15.22 \%$ of the phenotypic variation. Among them, the major QTL QGlo1B was detected at two environments and accounted for $14.12 \%$ and $15.21 \%$ of the phenotypic variation at each of these environments. The major QTL, $Q G l o 1 B$, was detected, with the largest contribution to the phenotypic variation $(15.22 \%)$.

Three conditional additive QTLs for globulin content were detected (Table S4; Fig. S1) and were mapped to chromosomes 2A, 6A and 6B. These QTLs each accounted for $7.04-9.12 \%$ of the phenotypic variation.

\section{Unconditional and conditional QTLs for gliadin content}

A total of six unconditional additive QTLs for gliadin content were identified within 2 years at three locations (Table S3; Fig. S1). These QTLs were mapped to chromosomes $1 \mathrm{~B}, 2 \mathrm{~A}, 4 \mathrm{~A}, 5 \mathrm{~A}, 7 \mathrm{~A}$ and $7 \mathrm{~B}$ and accounted for $6.67-15.00 \%$ of the phenotypic variation. Among them, $Q G l i 7 B$ was detected at two environments and accounted for $8.33 \%$ and $9.98 \%$ of the phenotypic variation at each of these environments. The major QTL, QGli1B, was detected, with the largest contribution to the phenotypic variation $(15.00 \%)$.

Five conditional additive QTLs for gliadin content were identified (Table S4; Fig. S1). These QTLs were mapped to chromosomes 1B, 2A, 4A, 7A and 7B and accounted for $6.42-15.00 \%$ of the phenotypic variation. Among them, QGli7B was detected at two environments and accounted for $7.022 \%$ and $8.233 \%$ of the phenotypic variation at each of these environments. The major QTL, QGli1B, was detected, with the largest contribution to the phenotypic variation $(15.00 \%)$.

\section{Unconditional and conditional QTLs for glutenin content}

Four different unconditional additive QTLs for glutenin content were identified within 2 years at three locations (Table S3; Fig. S1). These QTLs were mapped to chromosomes $1 \mathrm{~B}, 1 \mathrm{D}, 3 \mathrm{~A}$ and $3 \mathrm{~B}$ and accounted for $7.26-16.79 \%$ of the phenotypic variation. Among 
them, QGlu3A and $Q G l u 1 D$ were detected at two environments and accounted for 7.26$12.74 \%$ of the phenotypic variation. Two major QTLs, QGlu1B and QGlu1D, were detected, with $Q G l u 1 B$ having the largest contribution to phenotypic variation $(16.79 \%)$.

Five conditional additive QTLs for glutenin content were identified (Table S4; Fig. S1). These QTLs were mapped to chromosomes 1A, 1B, 1D and 3A and accounted for $6.20-16.86 \%$ of the phenotypic variation. Among them, $Q G l u 3 A$ and $Q G l u 1 D$ were detected at two environments and accounted for $6.20-12.70 \%$ of the phenotypic variation. Two major QTLs, QGlu1B and QGlu1D, were detected, with $Q G l u 1 B$ having the largest contribution to phenotypic variation $(16.86 \%)$.

\section{Discussion}

Wen and Zhu (2005) pioneered the use of conditional QTL analysis for complex traits and their components. The major advantage of this method is that it allows the determination of the net contribution of protein-fraction contents to starch content. In this study, we used both unconditional and conditional mapping and found that there were some QTLs, which were not detected using unconditional mapping, or which had different additive effects and contributions in the two types of mapping. Conditional mapping allowed us to pinpoint the reduction of the protein-fraction contents variances after excluding the influences of starch content, and the declined protein-fraction contents variances were even more obvious when protein-fraction contents was conditioned on starch content.

We evaluated the genetic relationships among protein-fraction contents and starch content by comparing the genetic effect of unconditional and conditional QTL. Four types of QTLs controlling protein-fraction contents were identified in this study. The first class of protein-fraction contents QTL had a similar or equal additive effect in both unconditional and conditional analyses, indicating that this type of QTL only controlled protein-fraction contents and not starch content. For example, QAlu1B (Alu/starch) associated with albumin content was independent of seed starch content.

This type of QTL is of special interest in wheat breeding as it would allow the increase of protein-fraction without a concomitant decrease in starch. Genes underlying this type of QTL are often involved in regulation of a trait (Liu et al. 2002; Song et al. 2007). Hence, this type of QTL as identified by conditional analyses would be valuable in selection of high-protein lines without reduction of starch content.

The second class of protein-fraction contents QTL was identified by either a greatly enhanced or reduced effect between unconditional and conditional analyses. This type of QTL is partially associated with starch content and could increase or decrease starch content. For example, QGli2A located within interval Xwmc177-Xgpw2321 decreased starch content. Although the unconditional additive effects of these loci might be relatively small and insignificant compared with genes that directly control starch content, the information would still be valuable for improving both seed protein-fraction contents and starch content.

The third class of QTL could be only detected by conditional analyses. For example, QAlu1B-2 (Alu/starch) for protein-fraction contents is conditional on starch content. This 
type of protein-fraction contents QTL was completely suppressed by starch content, and its additive effects could only be identified when the influence of starch content was eliminated. These QTL might have opposite additive effects between protein-fraction contents and starch content. If these QTL were used to modify protein-fraction contents, it could be difficult to simultaneously improve starch content.

The fourth class of protein-fraction contents QTL detected by the unconditional analyses was undetectable through conditional analyses, indicating that some loci underlying protein-fraction contents might be entirely due to starch content. For example, QAlu3A, located in Xwmc264-Xcfa2193, was identified for albumin content (Table S4).

Protein-fraction and starch are important factors for wheat quality and yield. Proteinfraction and starch contents in wheat grains are affected by genes and environment. To date, many studies carried out on QTLs in wheat have focused on protein-fraction content, which are distributed on at least 15 chromosomes, such as $1 \mathrm{~A}, 1 \mathrm{~B}, 2 \mathrm{~A}, 2 \mathrm{~B}, 2 \mathrm{D}$, 3A, 3B, 4A, 5B, 5D, 6B, 6D, 7A, 7B, and 7D chromosomes (Charmet et al. 2005; Mann et al. 2009; Merlino et al. 2009; Zhang et al. 2011). In the present study, atotal of 17 unconditional additive QTLs (four albumin QTLs, three globulin QTLs, six gliadin QTLs, four glutenin QTLs) controlling protein component content were detected. QTLs for several of our protein-fractions localized to the same chromosomes as had been reported in previous studies. For example, Shi et al. (2008) reported that the QTLs of albumin content were located on chromosome 3A and the QTLs of gliadin content were located on chromosome 1B. Zhang et al. (2011) have also identified the QTLs of glutenin content on chromosomes 1B, 1D and 3A.

Conditional additive QTLs controlling protein-fraction content were mapped. The 19 conditional QTLs (five albumin QTLs, three globulin QTLs, five gliadin QTLs, six glutenin QTLs) were detected in the DH population based on starch content. We identified some important QTLs for improving protein-fraction content. Among these QTLs, $Q A$ $l u 1 B$ increased albumin content and was unrelated to starch content; $Q G l o 6 A$ increased globulin content and did not reduce starch content, contra to the usual negative correlation between globulin content and starch content; similarly; QGli1B and QGli7A increased gliadin content and did not reduce starch content, contra to the usual negative correlation between gliadin content and starch content; QGlu1B and QGlu1D could increase glutenin content and did not reduce starch content, contra to the usual negative correlation between glutenin content and starch content. These QTLs might provide valuable information for marker-assisted selection to improve protein-fraction content without reducing starch content.

\section{Acknowledgements}

This study was supported by the Hebei Basic Key Research Program (13966302). The Fund for Team building of Crop Science Hebei Agricultural University (TD2016C3081). 


\section{References}

Branlard, G., Dardevet, M.1985. Diversity of grain protein and bread wheat quality: II. Correlation between high molecular weight subunits of glutenin and flour quality characteristics. J. Cereal Sci. 3:345-354.

Charmet, G., Robert, N., Branlard, G., Linossier, L., Martre, P., Triboï, E. 2005. Genetic analysis of dry matter and nitrogen accumulation and protein composition in wheat kernels. Theor. Appl. Genet. 111:540-550.

Cheng, X.X., Cheng, J.P., Huang, X., Lai, Y.Y., Wang, L., Du, W.L., Wang, Z.F., Zhang, H.S. 2013. Dynamic quantitative trait loci analysis of seed reserve utilization during three germination stages in rice. Plos One 8:e80002.

George, L., Scott, B. 1995. Separation and characterization of wheat protein fractions by high-performance capillary electrophoresis 1 . Cereal Chem. 72:527-532.

Guo, C.Q., Bai, Z.A., Liao, P.A., Jin, W.K. 2004. New high quality and yield wheat variety Yumai 57. China Seed Ind. 4:54.

Han, Y.P., Xie, D.W., Teng, W.L., Zhang, S.Z., Chang, W., Li, W.B. 2011. Dynamic QTL analysis of linolenic acid content in different developmental stages of soybean seed. Theor. Appl. Genet. 122:1481-1488.

Hai, Y., Kang, M.H. 2007. Breeding of a new wheat variety Huapei 3 with high yield and early maturity. Henan Agr. Sci. China 5:36-37.

Holm, J., Bjorck, I., Drews, A. 1986. A rapid method for the analysis of starch. Food Chem. 38:224-226.

Huebner, F.R., Wall, J.S. 1976. Fractionation and quantitative differences of glutenin from wheat varieties varying in baking quality. Cereal Chem. 53:258-269.

Hurkman, W.J., McCue, K.F., Altenbach, S.B., Korn, A., Tanaka, C.K., Kothari, K.M., Johnson, E.L., Bechtel, D.B., Wilson, J.D., Anderson, O.D., DuPont, F.M. 2003. Effect of temperature on expression of genes encoding enzymes for starch biosynthesis in developing wheat endosperm. Plant Sci. 164:873-881.

Jenner, C. 1994. Starch synthesis in the kernel of wheat under high temperature conditions. Funct. Plant Biol. 21:791-806.

Jiang, P., Wan, Z.Y., Wang, Z.X., Li, S.S., Sun, Q.Q. 2013. Dynamic QTL analysis for activity of antioxidant enzymes and malondialdehyde content in wheat seed during germination. Euphytica 190:75-85.

Jiang, Z.F., Han, Y.P., Teng, W.L., Zhang, Z.C., Sun, D.S., Li, Y.H., Li, W.B. 2010. Identification of QTL underlying the filling rate of protein at different developmental stages of soybean seed. Euphytica 175:227-236.

Lasztity, R. 1984. Wheat Proteins. The Chemistry of Cereal Proteins. Boca Raton, FL, USA. pp. 73-89.

Li, W.F., Liu, B., Peng, T., Yuan, Q.Q., Han, S.X., Tian, J.C. 2012. Detection of QTL for kernel weight, grain size, and grain hardness in wheat using $\mathrm{DH}$ and immortalized $\mathrm{F}_{2}$ population. Agric. Sci. China 45:34533462 .

Lian, X.J., Guo, J.J., Wang, D.L., Li, L., Zhu, J.R. 2014. Effects of protein in wheat flour on retrogradation of wheat starch. J. Food Sci. 79:1505-1511.

Liu, G., Xu, S.B., Ni, Z.F., Xie, C.J., Qin, D.D., Li, J., Lu, L.H., Zhang, J.P., Peng, H.R., Sun, Q.X. 2011. Molecular dissection of plant height QTLs using recombinant inbred lines from hybrids between common wheat (Triticum aestivum L.) and spelt wheat (Triticum spelta L.). Chinese Sci. Bull. 56:1897-1903.

Liu, G.F., Yang, J., Xu, H.M., Hayat, Y., Zhu, J. 2008. Genetic analysis of grain yield conditioned on its component traits in rice (Oryza sativa L.). Aust. J. Agr. Res. 59:180-195.

Liu, J.P., Van Eck, J., Cong, B., Tanksley, S.D. 2002. A new class of regulatory genes underlying the cause of pear-shaped tomato fruit. P. Natl Acad. Sci. USA 99:13302-13306.

MacRitchie, F. 1992. Physicochemical properties of wheat proteins in relation to functionality. Adv. Food Nutr. Res. 36:1-87.

Mann, G., Diffey, S., Cullis, B., Azanza, F., Martin, D., Kelly, A., McIntyre, L., Schmidt, A., Ma, W., Nath, Z., Kutty, I., Leyne, P.E., Rampling, L., Quail, K.J., Morell, M.K. 2009. Genetic control of wheat quality: interactions between chromosomal regions determining protein content and composition, dough rheology, and sponge and dough baking properties. Theor. Appl. Genet. 118:1519-1537.

Martin, J.M., Talbert, L.E, Habernicht, D.K., Lanning, S.P., Sherman, J.D., Carlson, G., Giroux, M.J. 2004. Reduced amylose effects on bread and white salted noodle quality. Cereal Chem. 81:188-193.

McIntosh, R.A., Hart, G.E., Gale, M.D. 1994. Catalogue of gene symbols for wheat. Wheat Inf. Serv. 79:47-56. 
Merlino, M., Leroy, P., Chambon, C., Branlard, G. 2009. Mapping and proteomic analysis of albumin and globulin proteins in hexaploid wheat kernels (Triticum aestivum L.). Theor. Appl. Genet. 118:1321-1337.

Osborne, T.B. 1907. The Protein of the Wheat Kernel. Publication of the Carnegie Institute. Washington, USA.

Osman, K.A., Tang, B., Wang, Y.P., Chen, J.H., Yu, F., Liu, L., Han, X.S., Zhang, Z.X., Yan, J.B., Zheng, Y.L., Yue, B., Qiu, F.Z. 2013. Dynamic QTL analysis and candidate gene mapping for waterlogging tolerance at maize seedling stage. Plos One 8:e79305.

Pence, J.W., Weinstein, N.E., Mecham, D.K. 1954. The albumin and globulin contents of wheat flour and their relationship to protein quality. Cereal Chem. 31:303-311.

Pike, P.R., MacRitchie, F. 2004. Protein composition and quality of some new hard white winter wheats. Crop Sci. 44:173-176.

Shi, P.C., Cao, L.P., Wang, G.L., Zhang, W. 2008. Mapping QTLs related to protein components of grain of common wheat. J. Trit. Crops 28:550-554.

Singh, J., Skerritt, J.H. 2001. Chromosomal control of albumins and globulins in wheat grain assessed using different fractionation procedures. J. Cereal Sci. 33:163-181.

Somers, D.J., Isaac, P., Edwards, K. 2004. A high-density microsatellite consensus map for bread wheat (Triticum aestivum L.). Theor. Appl. Genet. 109:1105-1114.

Song, X.J., Huang, W., Shi, M., Zhu, M.Z., Lin, H.X. 2007. A QTL for rice grain width and weight encodes a previously unknown RING-type E3 ubiquitin ligase. Nat. Genet. 39:623-630.

Wang, L., Cui, F., Wang, J.P., Jun, L., Ding, A.M., Zhao, C.N., Li, X.F., Feng, D.S., Gao, J.R., Wang, H.G. 2012. Conditional QTL mapping of protein content in wheat with respect to grain yield and its components. J. Genet. 91:303-312.

Weegels, P.L., Hamer, R.J., Schofield, J.D. 1996. Functional properties of wheat glutenin. J. Cereal Sci. 23: $1-18$.

Wen, Y.X., Zhu, J. 2005. Multivariable conditional analysis for complex trait and its components. Acta Genet. Sin. 32:289-296.

Wieser, H. 2007. Chemistry of gluten proteins. Food Microbiol. 24:115-119.

Yamamori, M., Quynh, N.T. 2003. Diversity effects of Wx-A1, -B1 and -D1 protein deficiencies on apparent amylose content and starch pasting properties in common wheat. Theor. Appl. Genet. 100:32-38.

Yamamori, M. 2009. Amylose content and starch properties generated by five variant Wx alleles for granulebound starch synthase in common wheat (Triticum aestivum L.). Euphytica 165:607-614.

Ye, Z.H., Lu, Z.Z., Zhu, J. 2003. Genetic analysis for developmental behavior of some seed quality traits in upland cotton (Gossypum hirsutum L.). Euphytica 129:183-191.

Zhang, K.P., Zhao, L., Tian, J.C., Chen, G.F., Jiang, X.L., Liu, B. 2008. A genetic map constructed using a doubled haploid population derived from two elite Chinese common wheat varieties. J. Integr. Plant Biol. 50:941-950.

Zhang, Y., Tang, J.W., Yan, J., Zhang, Y.L., Zhang, Y., Xia, X.C., He, Z.H. 2009. The gluten protein and interactions between components determine mixograph properties in an $\mathrm{F}_{6}$ recombinant inbred line population in bread wheat. J. Cereal Sci. 50:219-226.

Zhang, Y., Tang, J.W., Zhang, Y.L., Yan, J., Xiao, Y.G., Zhang, Y., Xia, X.C., He, Z.H. 2011. QTL mapping for quantities of protein fractions in bread wheat (Triticum aestivum L.). Theor. Appl. Genet. 122:971-987.

Zhao, J.Y., Becker, H.C., Zhang, D.Q., Zhang, Y.F., Ecke, W. 2006. Conditional QTL mapping of oil content in rapeseed with respect to protein content and traits related to plant development and grain yield. Theor. Appl. Genet. 113:33-38.

Zhao, L., Zhang, K.P., Liu, B., Deng, Z.Y., Qu, H.L., Tian, J.C. 2010. A comparison of grain protein content QTLs and flour protein content QTLs across environments in cultivated wheat. Euphytica 174:325-335.

Zhu, J. 1995. Analysis of conditional genetic effects and variance components in developmental genetics. Genetics 141:1633-1639.

Zhu, Z.L., Liu, B., Tian, B., Xie, Q.G., Li, W.F., Tian, J.C. 2011. Dynamic QTL mapping of wheat protein content in developing grains. Agr. Sci. China 44:3078-3085. 


\section{Electronic Supplementary Material (ESM)}

Electronic Supplementary Material (ESM) associated with this article can be found at the website of CRC at http://www.akademiai.com/content/120427/

Electronic Supplementary Table S1. Statistical analysis of starch content and protein-fraction contents in parents Huapei 3 (HP3) and Yumai 57 (YM57) and the doubled haploid (DH) population in six environments (\%)

Electronic Supplementary Table S2. Relationship coefficients between protein-fraction contents and starch content in six environments

Electronic Supplementary Table S3. Identification of unconditional QTLs with additive effects for proteinfraction contents in DH population over six environments

Electronic Supplementary Table S4. Identification of conditional QTLs with additive effects for protein-fraction contents in DH population over six environments

Electronic Supplementary Figure S1. The unconditional and conditional QTLs controlling the protent-fraction content of wheat at different environment 